\title{
Primary resistance phenomena to oncolytic measles vaccine viruses
}

\author{
MARKUS NOLL ${ }^{1}$, SUSANNE BERCHTOLD ${ }^{1}$, JOHANNA LAMPE ${ }^{1,2}$, NISAR P. MALEK ${ }^{1}$, \\ MICHAEL BITZER ${ }^{1}$ and ULRICH M. LAUER ${ }^{1}$ \\ ${ }^{1}$ Department of Internal Medicine I, University Hospital Tübingen, D-72076 Tübingen, Germany
}

Received February 5, 2013; Accepted March 22, 2013

DOI: 10.3892/ijo.2013.1914

\begin{abstract}
Measles vaccine virus (MeV) has been shown to possess profound oncolytic capabilities. However, tumor cell resistance to $\mathrm{MeV}$ may endanger broad clinical success. Here, this hypothesis is underlined by our analysis of the NCI-60 tumor cell panel infected with a suicide gene-armed $\mathrm{MeV}$ vector (MeV-SCD). Quantification of the MeV-SCD-induced oncolytic effect exhibited a 50\% rate of NCI-60 solid tumor cell lines being susceptible to MeV-SCD induced oncolysis. In contrast, nearly $40 \%$ of the NCI-60 tumor cell lines had to be categorized as partially resistant (exhibiting 50-75\% remnant
\end{abstract}

Correspondence to: Professor Ulrich M. Lauer, Department of Internal Medicine I, University Hospital Tübingen, Otfried-Müller-Str. 10, D-72076 Tübingen, Germany

E-mail: ulrich.lauer@uni-tuebingen.de

Present address: ${ }^{2}$ Institute for Molecular Medicine Finland FIMM, Helsinki, Finland

Abbreviations: 5-FC, 5-fluorocytosine; 5-FU, 5-fluorouracil; 5-FUMP, 5-fluorouridine monophosphate; CEA, carcinoembryonic antigen; DMEM, Dulbecco's modified Eagle's medium; eIF, eukaryotic initiation factor; FCS, fetal calf serum; GFP, green fluorescent protein; hpi, hours post infection; h, hours; IFIT, interferon-induced protein with tetratricopeptide repeats; IFN, interferon; IFNAR, interferon- $\alpha / \beta$ receptor; IRF, interferon regulatory factor; ISG, interferon stimulated gene; ISRE, interferon stimulated response element; Jak, Janus kinase; MDA, melanoma differentiation-associated gene; $\mathrm{MeV}$, measles vaccine virus; MOI, multiplicity of infection; N, nucleocapsid protein; NCI, US National Cancer Institute; NF- $\kappa \mathrm{B}$, nuclear factor $\kappa$-light chain-enhancer of activated B cells; PBS, phosphate-buffered saline; PAGE, polyacrylamide gel electrophoresis; PFA, paraformaldehyde; PFU, plaque-forming units; PVDF, polyvinylidene difluoride; RIG, retinoic acid inducible gene; RT, room temperature; SCD, super cytosine deaminase; SD, standard deviation; SDS, sodium dodecyl sulfate; SEM, standard error of the mean; SRB, sulforhodamine B; STAT, signal transducers and activators of transcription; TBS-T, Trisbuffered saline containing $0.02 \%$ Tween-20; TCA, trichloroacetic acid

Key words: oncolysis, measles vaccine virus, oncolytic virotherapy, resistance to virotherapy, interferon signaling tumor cells) and six tumor cell lines even showed high resistance to MeV-SCD-induced oncolysis with remnant tumor cell masses $>75 \%$. According to our further analysis, these high-grade resistant tumor cell lines i) exhibited a high variation in primary infectability rates and also different patterns of alterations ii) in virus replication and iii) in interferon response. This diversity of virotherapy resistance phenomena seems to go along with the diversity of genetic and epigenetic changes accompanying malignant transformation. Of paramount clinical importance, this plethora of resistance phenomena was shown to be overcome in vitro by employment of an increased MOI together with addition of the prodrug 5-FC, thus exploiting the highly efficient suicide gene function of vector MeV-SCD used in this study.

\section{Introduction}

Since early observations of tumor lytic effects in the course of natural virus infections (1-5), many different virus types have been characterized in detail and were found to exhibit profound oncolytic capacities (6). Among those, measles vaccine virus $(\mathrm{MeV})$ constitutes a leading vector type and has shown its oncolytic potential for example in ovarian cancer, not only in extensive pre-clinical examinations (7), but also in a recent clinical study (8). However, a detailed analysis of the clinical data revealed that i) $\mathrm{MeV}$ infected only few ovarian cancer cells and, importantly, ii) was not substantially amplified in the tumor cells of the respective ovarian cancer patients (9). Since this clinical outcome is significantly inferior compared with results obtained in rodents, it seems to be quite essential to characterize and unravel the underlying mechanisms of primary resistance to virotherapy in general and to develop broadly applicable approaches which are effective in overcoming these limitations in different cancer entities.

In this study, we screened 54 cell lines of the NCI-60 tumor cell panel [representing colon, CNS, lung, mammary, melanoma, ovarian, prostate and renal cancers (10)] for different mechanisms of resistance to our suicide gene armed virotherapeutic vector MeV-SCD, which codes for a fusion protein of yeast cytosine deaminase and yeast uracil phosphoribosyltransferase that converts the prodrug 5-fluorocytosine (5-FC) into the well-known chemotherapeutic 5-fluorouracil (5-FU) and facilitates further conversion into the cytotoxic compound 5-fluorouridine monophosphate (5-FUMP) (11). 
First, we proved sufficient expression of the CD46 receptor for the NCI-60 tumor cell panel which is needed for MeV-induced intercellular fusion/syncytium formation and subsequent $\mathrm{MeV}$-mediated oncolysis (12). After classifying all 54 cell lines into different levels of resistance to MeV-based oncolysis, we specifically analyzed the high grade resistant cell lines for their primary infection rates, their expression of $\mathrm{MeV}$-encoded proteins, their patterns of alterations in $\mathrm{MeV}$ replication and in interferon response. Furthermore, in order to determine the overall feasibility of a MeV-SCD based virotherapy in clinical practice, we also evaluated its oncolytic effect i) in presence of the prodrug 5-FC and ii) with or without usage of an increased MeV-SCD virus load.

\section{Material and methods}

Cell culture. The US National Cancer Institute's NCI-60 tumor cell panel was purchased from Charles River Laboratories (Charles River Laboratories Inc., New York, NY, USA). African green monkey kidney (Vero) cells were obtained from the German Collection of Microorganisms and Cell Cultures (DSMZ, Braunschweig, Germany). All NCI-60 cell lines were grown in RPMI-1640 (PAA Laboratories, Pasching, Austria) growth medium, Vero cells were grown in Dulbecco's modified Eagle's medium (DMEM, Biochrom, Berlin, Germany). Medium was supplemented with $10 \%$ fetal calf serum (FCS, PAA Laboratories). Cells were cultivated at $37^{\circ} \mathrm{C}$ in a humidified atmosphere containing $5 \% \mathrm{CO}_{2}$.

Production and titration of measles vaccine virus. Vero cells $\left(1 \times 10^{7}\right)$ were seeded in $15-\mathrm{cm}$ plates (Greiner BioOne, Frickenhausen, Germany). The next day, cells were washed with phosphate-buffered saline (PBS, PAA Laboratories) and infected at a multiplicity of infection (MOI) of 0.03 in Opti-MEM (Invitrogen, Carlsbad, CA, USA) for $3 \mathrm{~h}$. Then, medium was replaced with DMEM supplemented with $10 \%$ FCS. Fifty-four hours later, when most of the cells were infected, medium was removed, cells were scraped into $1 \mathrm{ml}$ Opti-MEM and lysed by freeze/thaw. After centrifugation $\left(1,900 \mathrm{x} \mathrm{g}, 15 \mathrm{~min}, 4^{\circ} \mathrm{C}\right)$, the supernatant was stored at $-80^{\circ} \mathrm{C}$. Viral titers were determined according to the method of Kärber (13) and Spearman (14) on Vero cells as described earlier. Infected cells were detected by immunofluorescence staining. Cells were washed with PBS and fixed with $4 \%$ PFA for $10 \mathrm{~min}$ at room temperature (RT). After washing twice with PBS and blocking for 30 min with $1 \%$ FCS in Tris-buffered saline containing $0.02 \%$ Tween-20 (TBS-T), cells were incubated with anti-measles $\mathrm{N}$ protein antibody (European Collection of Cell Cultures, ECACC, Salisbury, UK, clone 120, no. 95040312, 1:1,000) for $30 \mathrm{~min}$ at RT. After washing three times with TBS-T, incubation with a goat anti-mouse secondary antibody (Invitrogen, Alexa Fluor 546, 1:1,000) was performed for $30 \mathrm{~min}$ in the dark. Cells were washed again three times with TBS-T and analyzed by fluorescence microscopy (IX50, Olympus, Tokyo, Japan; analySIS, Soft Imaging System, Münster, Germany).

Detection of CD46 expression by flow cytometry. Cells were washed with PBS, detached with accutase (PAA Laboratories) and diluted in FACS buffer (PBS containing 10\% FCS). Cells $\left(5 \times 10^{5}\right)$ were incubated with a phycoerythrin (PE)-labeled anti-human CD46 antibody (eBioscience Inc., San Diego, CA, USA) or a PE-labeled IgG1 mouse isotype control (eBioscience) diluted 1:20 in FACS buffer for $30 \mathrm{~min}$ on ice. Cells were washed with PBS, centrifuged for $5 \mathrm{~min}$ at $472 \mathrm{x}$ g and diluted in FACS buffer. If analysis was performed the next day, cells were fixed with $1.3 \%$ paraformaldehyde (Otto Fischar, Saarbruecken, Germany). Flow cytometry analysis was performed on FACSCalibur (Becton-Dickinson, Franklin Lakes, NJ, USA) using Cell Quest software (Becton-Dickinson). Mean fluorescence index ratio (12) is defined as the arithmetic mean of CD46 divided by the arithmetic mean of isotype control.

Sulforhodamine B (SRB) cell viability assay. A total of $4 \times 10^{4}$ cells/well in a 24-well plate were infected with MeV-SCD in $250 \mu \mathrm{l}$ Opti-MEM (Invitrogen, Darmstadt, Germany). At $3 \mathrm{~h}$ post infection (hpi) the inoculum was removed and growth medium with or without $1 \mathrm{mM}$ of the prodrug 5-FC (Roche, Grenzach-Wyhlen, Germany) was added to the cells.

As described previously (15), cells were washed with ice-cold PBS 96 hpi, fixed with cold 10\% trichloroacetic acid (TCA) and incubated at $4^{\circ} \mathrm{C}$ for $30 \mathrm{~min}$. After removing TCA and drying $0.4 \%$ (wt/vol) sulforhodamine B (SRB) (Sigma-Aldrich, Taufkirchen, Germany) dissolved in $1 \%$ acetic acid were added and cells were stained at RT for $10 \mathrm{~min}$.

After washing with $1 \%$ acetic acid and drying, $10 \mathrm{mM}$ Tris base $(\mathrm{pH} 10.5)$ was added in order to extract protein-bound dye and incubated at RT for $10 \mathrm{~min}$. Optical density was measured in a microtiter plate reader (Tecan Genios Plus, Tecan Deutschland, Crailsheim, Germany) at a wavelength of $550 \mathrm{~nm}$ (reference wavelength at $620 \mathrm{~nm}$ ).

Quantification of primary infection. A total of $3 \times 10^{5}$ cells/well were seeded in a 6-well plate. Infection with MeV-GFP [green fluorescent protein (GFP)-encoding $\mathrm{MeV}$ vector in which the GFP marker gene is placed at the same position as the SCD suicide gene in MeV-SCD] was performed in $1 \mathrm{ml}$ Opti-MEM per well. Infection medium (3 hpi) was replaced by $2 \mathrm{ml}$ standard growth medium. At 24 hpi, cells were washed with PBS, detached with accutase (PAA Laboratories) and resuspended in $1 \mathrm{ml}$ FACS buffer and $3 \mathrm{ml} \mathrm{PBS}$. After centrifugation (5 min at $302 \times \mathrm{g}$ ) at RT the pellet was resuspended in FACS buffer. Cells were fixed with $1.3 \%$ paraformaldehyde (PFA, Fischar) and analyzed on FACSCalibur (Becton-Dickinson) using the Cell Quest software (Becton-Dickinson).

Virus growth curve. Cells $\left(1 \times 10^{5}\right.$ cells/well in a 6 -well plate) were infected with MeV-SCD in $1 \mathrm{ml}$ Opti-MEM. At 3 hpi, medium was removed and cells were washed three times with

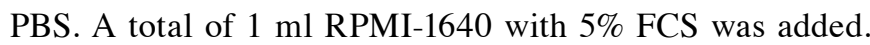
Supernatants and cells (scraped into $1 \mathrm{ml}$ Opti-MEM) were harvested at 3, 24, 48, 72 and 96 hpi and subjected to one freeze-thaw cycle. Cell-associated and released virus were quantified as described above.

Immunoblot analysis. Cells $\left(1 \times 10^{6}\right)$ were seeded in a $60-\mathrm{mm}$ tissue culture dish. Twenty-four hours later, cells were infected with $\mathrm{MeV}-\mathrm{SCD}$ in $2 \mathrm{ml}$ Opti-MEM. At $3 \mathrm{hpi}$, the inoculum was replaced by standard growth medium. At the indicated time points cells were washed with PBS and lysed in lysis buffer (50 mM Tris, $150 \mathrm{mM} \mathrm{NaCl}, 1 \%$ Nonidet P-40). Lysates were 
then subjected to three freeze-thaw cycles and centrifuged. Protein concentration in the supernatants was determined by Bradford protein assay (Bio-Rad, Hercules, CA, USA). A total of $50 \mu \mathrm{g}$ (MeV N-protein and SCD) or $75 \mu \mathrm{g}$ (Stat1, P-Stat1, IFIT1) protein were diluted in 6-fold Roti Load Buffer (Carl Roth, Karlsruhe, Germany) and denatured for $5 \mathrm{~min}$ at $95^{\circ} \mathrm{C}$. Proteins were separated by $8 \%$ SDS-PAGE and transferred to a hydrophobic polyvinylidene difluoride (PVDF) membrane (Hybond-P, GE Healthcare, Waukesha, WI, USA). After blocking with 5\% powdered milk (Carl Roth) in Trisbuffered saline containing $0.02 \%$ Tween-20 (TBS-T) membranes were incubated with primary antibodies (anti-measles N-protein, ab23974, Abcam, Cambridge, UK, 1:6,000; anti-SCD, kind gift from Transgene S.A., Illkirch-Graffenstaden, France, 1:2,000; anti-P-Stat1, Cell Signaling Technology, \#9171, Danvers, MA, USA, 1:1,000; anti-Stat1, Santa Cruz Biotechnology, Santa Cruz, CA, USA, sc-592, 1:1,000; anti-IFIT1, GeneTex, Irvine, CA, USA, GTX103452, 1:1,000; anti-vinculin, Sigma-Aldrich, 1:5,000) with gentle shaking overnight at $4^{\circ} \mathrm{C}$. Membranes were washed three times with TBS-T, secondary antibodies [peroxidase-conjugated anti-rabbit (Bio-Rad, 1:5,000), anti-rat (Chemicon, Billerica, MA, USA, 1:8,000) or anti-mouse (BioRad, 1:8,000)] were added for $1 \mathrm{~h}$ and the membranes were washed another three times with TBS-T. Proteins were detected with Amersham ECL Western Blotting Detection Reagents (GE Healthcare). A prestained protein ladder (PageRuler Plus, Thermo-Scientific, Waltham, MA, USA) was used for determination of molecular weight.

Statistical analysis. Results of cell mass determination after infection with MeV-SCD are expressed as mean \pm standard deviation (SD) or as mean \pm standard error of the mean (SEM) as indicated. Results of viral growth curves are expressed as mean \pm SD. Statistical analysis was done by one-way analysis of variance (ANOVA) followed by Bonferroni's Multiple Comparison Test. Results were calculated using GraphPad Prism Software version 4.03 (GraphPad Software, La Jolla, CA, USA). P-values $<0.05$ were regarded as statistically significant.

\section{Results}

Screening the NCI-60 tumor panel for resistances to oncolysis with $\mathrm{MeV}$-SCD. In order to detect possible resistances against $\mathrm{MeV}$-induced oncolysis, cell lines taken from the well-classified NCI-60 tumor cell panel (10) were infected with a suicide genearmed oncolytic measles vaccine virus vector, $\mathrm{MeV}-\mathrm{SCD}$ (11). Due to our focus on solid tumors, six cell lines originating from leukemias were omitted, leaving a panel of 54 cell lines of true solid tumor origin for our study, representing eight tumor types: colon ( $n=7)$, CNS ( $n=6)$, lung $(n=9)$, mammary $(n=6)$, melanoma $(n=9)$, ovarian $(n=7)$, prostate $(n=2)$ and renal $(n=8)$ tumors.

At first, infection with MeV-SCD was performed without addition of the prodrug 5-FC under standardized conditions using a multiplicity of infection (MOI) of 1 . At $96 \mathrm{~h}$ post infection (hpi), the remaining tumor cell mass was determined using the SRB cytotoxicity assay, known as the US National Cancer Institute's (NCI) standard assay for measuring the cellular protein content after incubation with cytotoxic substances (15) which constitutes an equivalent of tumor cell masses remaining as a result of cytotoxic treatment.
Depending on their remaining tumor cell mass under treatment with $\mathrm{MeV}-\mathrm{SCD}$, cell lines exhibiting i) a remnant tumor cell mass $>75 \%$ were classified as high grade resistant tumor cell lines, cell lines ii) with $50-75 \%$ remaining tumor cell mass were designated as partially resistant tumor cell lines, and cell lines iii) exhibiting a remaining tumor cell mass $<50 \%$ were referred to as tumor cell lines being susceptible to $\mathrm{MeV}$-SCD-induced oncolysis.

As a result, 27 of the 54 cell lines tested (i.e., 50\%) were classified as susceptible to MeV-SCD-mediated oncolysis (Fig. 1A; grey bars), i.e. loss of cell mass was $>50 \%$; the average remnant cell mass in this category at 96 hpi was $30.93 \%$. Furthermore, 21 cell lines (i.e., 39\%) proved to be partially resistant to MeV-SCD (Fig. 1A; yellow bars), with an average remnant cell mass of $62.08 \%$. Of note, six cell lines (i.e., $11 \%$ ) did categorize as being high grade resistant to MeV-SCD-induced oncolysis (Fig. 1A; red bars), manifesting an average remnant cell mass of $84.79 \%$.

The remaining cell masses of these six highly resistant tumor cell lines were as follows (see also Fig. 1A, bars from left to right): HCT-15 (adenocarcinoma of the colon), $98.41 \%$; KM12 (adenocarcinoma of the colon), 86.91\%; M14 (melanoma), 84.30\%; HOP-62 (adenocarcinoma of the lung), 83.74\%; ACHN (renal cell carcinoma), 79.79\%; and LOX-IMVI (malignant amelanotic melanoma), $75.59 \%$.

Interestingly, addition of the prodrug 5-FC at 3 hpi enhanced the oncolytic effect of MeV-SCD in a pronounced manner so that the whole panel of 54 tumor cell lines exhibited a remaining cell mass of $<50 \%$ (Fig. 1B; accordingly, all bars are now depicted in grey). Sticking to the classification named above, the average remaining cell mass of the 27 'already' susceptible cell lines declined to now only $7.41 \%$. The 21 formerly partially resistant cell lines now showed an average remnant cell mass of only $24.12 \%$ and the six formerly high grade resistant cell lines exhibited an average cell mass of now only $15.88 \%$. In detail, cell masses of former high grade resistant cell lines were now $34.54 \%$ for HCT-15, 5.98\% for KM12, 24.12\% for M14, $13.50 \%$ for HOP-62, $14.62 \%$ for ACHN, and $2.51 \%$ for LOX-IMVI (see also Fig. 1B, bars from left to right).

Based on these results on MeV-SCD-induced oncolysis, all following experiments were performed only with the high grade resistant tumor cell lines HCT-15, KM12, M14, HOP-62, ACHN and one control cell line, SW-620 (adenocarcinoma of the colon), classified as being susceptible to MeV-SCD oncolysis (remaining tumor cell mass at $96 \mathrm{hpi} / \mathrm{MOI} 1$ without 5-FC: $34.33 \%$; with addition of prodrug: $3.69 \%$ ).

High grade oncolysis resistant cell lines show a high variation in primary infection rates. To elucidate the mechanism of high grade resistance to $\mathrm{MeV}$-mediated oncolysis, we first confirmed high expression levels of the measles vaccine virus receptor CD46 on all tumor cell lines (data not shown). In order to further investigate if resistance was due to low primary infection rates, the cell lines were infected with a GFP marker gene expressing oncolytic measles vaccine virus vector, MeV-GFP, at MOI 0.1, 1, 10, or mock infected (Fig. 2). At 24 hpi, the percentage of GFP-expressing cells was quantified by flow cytometry.

At MOI 1, primary infection rates of the high grade resistant cell lines as given by the percentages of GFP-positive 


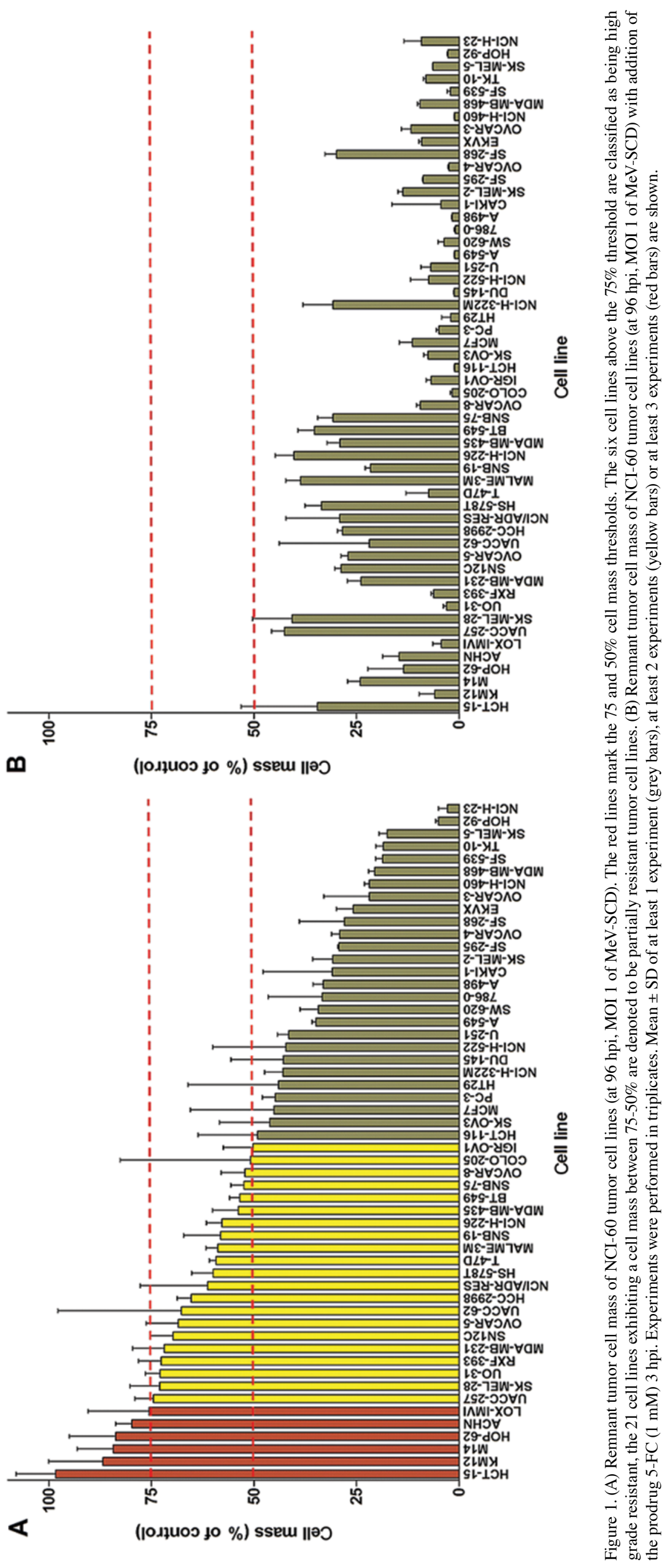




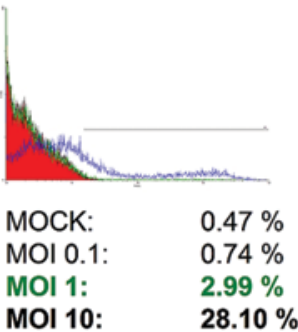

$\underline{\text { KM12 }}$
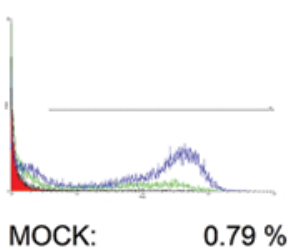

MOI 0.1: $\quad 2.96 \%$

MOI 1: $\quad 21.20 \%$

MOI 10: $\quad \mathbf{7 2 . 3 8 \%}$ $\underline{\text { M14 }}$

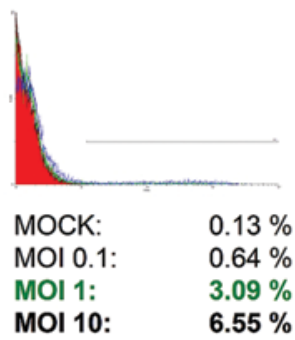

MOI 10: $\quad 6.55 \%$

$\underline{\mathrm{ACHN}}$

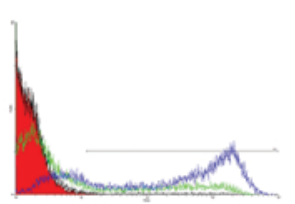

MOCK: $\quad 0.21 \%$

MOI 0.1: $\quad 2.99 \%$

MOI 1: $\quad 26.44 \%$

MOI 10: $\quad 73.73 \%$
HOP-62

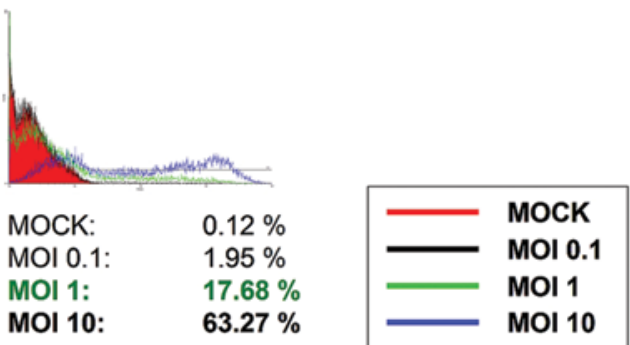

Figure 2. Primary infection rates of high grade MeV-SCD resistant tumor cell lines and one susceptible control tumor cell line. Cell lines were infected with MeV-GFP at MOI 0.1, 1 and 10; at 24 hpi, the percentage of GFP expressing cells was quantified by flow cytometry. Representatives of at least 2 experiments are shown.

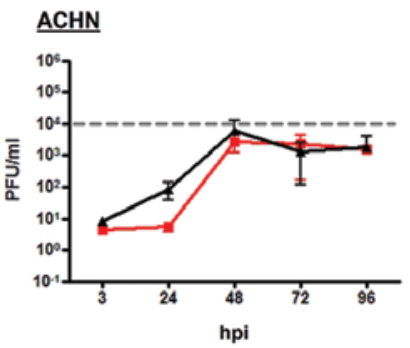

HCT-15
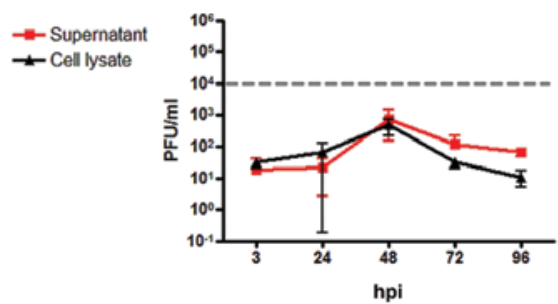

- - Supernatant $\leftarrow$ Cell lysate

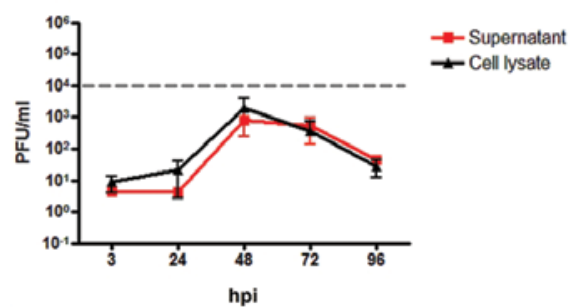

Control: MeV-SCD susceptible tumor cell line
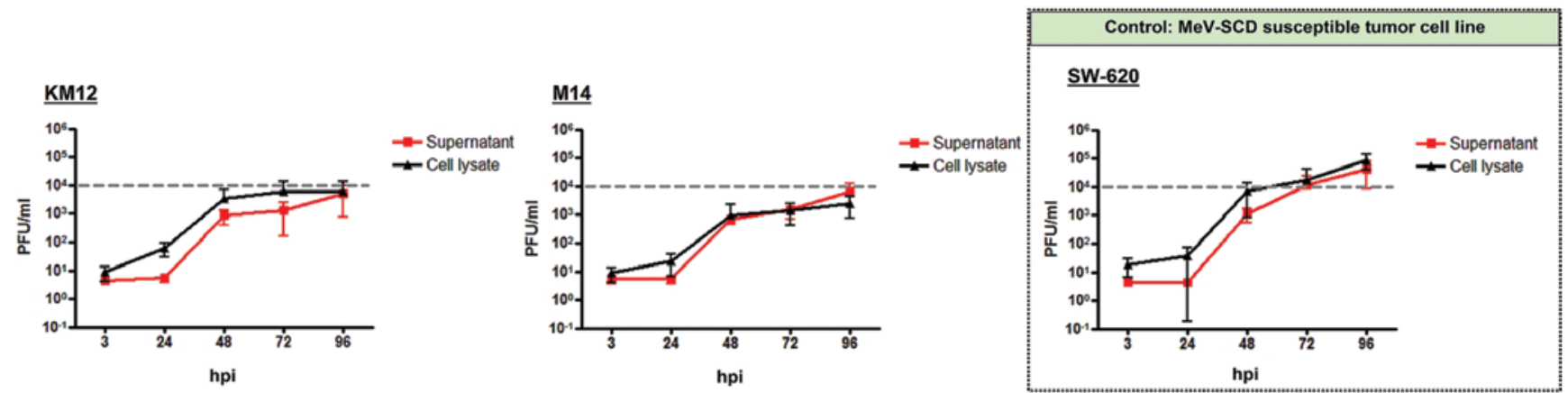

Figure 3. Viral growth curves showing quantification of viral replication in the selected tumor cell lines. All tumor cell lines were infected with MeV-SCD at MOI 0.03, except for HCT-15 (MOI 0.3). Supernatants and cells were harvested at the indicated time points. Titration was performed on Vero cells. Dotted lines in grey mark $10^{4}$ plaque-forming units $(\mathrm{PFU}) / \mathrm{ml}$. Mean $\pm \mathrm{SD}$ of three experiments are shown.

tumor cells varied between 2.99 and $26.44 \%$ (HCT-15, 2.99\%; M14, 3.09\%; HOP-62, 17.68\%; KM12, 21.20\%; and ACHN, $26.44 \%$, respectively), while the susceptible control cell line SW-620 exhibited a primary infection rate of $45.17 \%$.
Primary infection rates of the high grade resistant tumor cell lines at MOI 10 ranged from a minimum of 6.55 to a maximum of $73.73 \%$ (HCT-15, 28.10\%; M14, 6.55\%; HOP-62, 63.27\%; KM12, 72.38\%; and ACHN, 73.73\%, respectively), 
$\underline{\mathrm{ACHN}}$

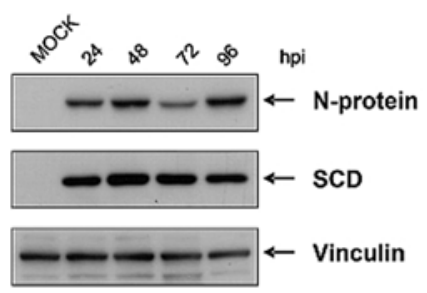

$\underline{\mathrm{KM} 12}$

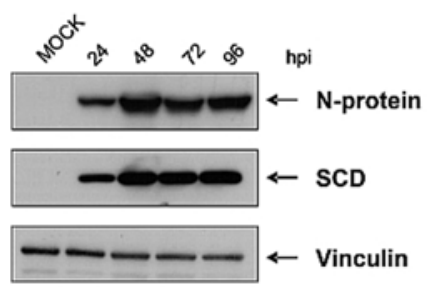

$\underline{\text { HCT-15 }}$

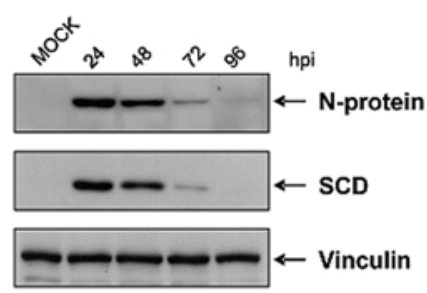

$\underline{\text { M14 }}$

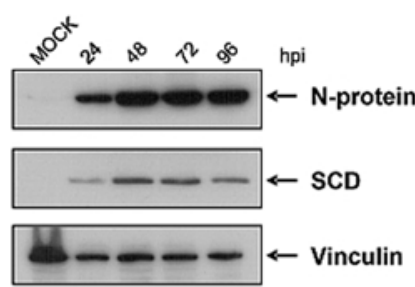

HOP-62

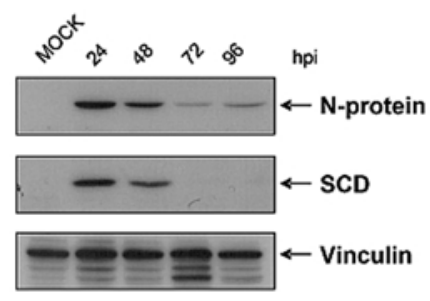

Control: MeV-SCD susceptible tumor cell line

$\underline{\text { SW-620 }}$

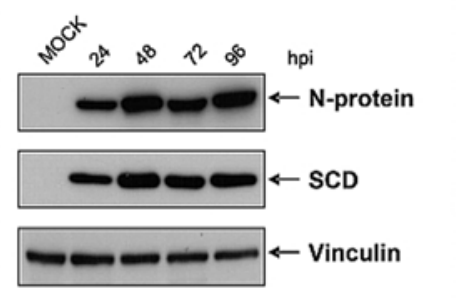

Figure 4. Expression of the MeV N protein and the SCD protein in the selected cell lines. Cells were infected with MeV-SCD at MOI 1 or mock infected. At the indicated time-points whole cell lysates were prepared. Expression of the MeV N-protein and the SCD protein was determined by immunoblot analysis. Vinculin was used as a loading control.

whereas the susceptible control cell line SW-620 showed a primary infection rate of $94.72 \%$.

In summary, primary infection rates were much lower in the high grade resistant cell lines when compared to the $\mathrm{MeV}$-oncolysis susceptible control cell line (SW-620). Notably, among the resistant cell lines there was a high degree of variation in primary infection rates ranging from 2.99 to $26.44 \%$ (MOI 1) and from 6.55 to $73.73 \%$ (MOI 10).

High grade resistant tumor cell lines show different patterns of altered $\mathrm{MeV}$ growth. To investigate viral replication, viral growth curves were generated with the five high grade resistant tumor cell lines and the susceptible tumor cell line (Fig. 3). For this purpose, tumor cell lines were infected with MeV-SCD at MOI 0.03; only HCT-15 cells were infected at a higher MOI of 0.3 because they were found to barely show any signs of viral growth at lower MOIs (data not shown).

While the control tumor cell line SW-620 showed an increase in viral titers of up to $10^{5}$ plaque-forming units $(\mathrm{PFU}) / \mathrm{ml}$, high grade resistant cell lines only reached titers below $10^{4} \mathrm{PFU} / \mathrm{ml}$ (indicated by the grey dotted lines in Fig. 3). Furthermore, different patterns of alterations in viral growth were found. In ACHN, KM12 and M14 tumor cells viral replication was reduced and reached a plateau as early as $48 \mathrm{hpi}$, indicating a reduced viral growth. In HCT-15 and HOP-62 tumor cells viral replication reached a peak at $48 \mathrm{hpi}$ and declined from that point on, reflecting a pattern of only transient viral growth.

$\mathrm{MeV}$-encoded proteins are expressed in all tested tumor cell lines. To prove the expression of virus-encoded proteins, high grade resistant tumor cell lines were infected with MeV-SCD at MOI 1 or mock infected. Cell lysates were prepared at 24, 48,
72 and 96 hpi. Western blot analysis confirmed expression of the $\mathrm{N}$-protein of $\mathrm{MeV}$ and of the vector-encoded suicide protein SCD in all tested tumor cell lines at 24 and 48 hpi (Fig. 4). High grade resistant tumor cell lines ACHN, KM12, M14 and the susceptible control tumor cell line SW-620 exhibited expression of N-protein and SCD at all time points. In contrast, cell lines HCT-15 and HOP-62 showed a decreased expression at 72 and 96 hpi (Fig. 4), which corresponds to the transient viral growth pattern observed in these two tumor cell lines (Fig. 3).

Alterations in interferon response of selected tumor cell lines. We next investigated if differences in interferon (IFN) signaling account for the differential susceptibility of tumor cell lines to $\mathrm{MeV}$-mediated oncolysis. IFN response induced by infection with MeV-SCD was examined by quantifying the expression of phosphorylated Stat1, total Stat1, and of the interferon stimulated gene IFIT1 (ISG56) via immunoblot analysis (Fig. 5). All tumor cell lines were infected with MeV-SCD at MOI 1 or mock infected and lysed at 24, 48 and $72 \mathrm{hpi}$.

As a result, the tested tumor cell lines exhibited different patterns in IFN response. Two tumor cell lines, i.e. the susceptible cell line SW-620 and the high grade resistant cell line KM12, showed no IFN response at all (Fig. 5, no signals for phosphorylated Stat1 and IFIT1). In contrast, cell lines ACHN, HCT-15, M14 and HOP-62 showed a strong expression of IFIT1 at 48 and 72 hpi. Interestingly, cell line HOP-62 only exhibited a transient phosphorylation of Stat 1 at 24 and 48 hpi. In ACHN cells, phosphorylation of Stat 1 was very weak. Finally, unphosphorylated Stat1 was found to be expressed in all tested cell lines (mock, as well as at 24, 48 and $72 \mathrm{hpi}$ ). Thus, different alterations in IFN response patterns were observed in the distinct tumor cell lines of the NCI-60 tumor cell panel. 
ACHN

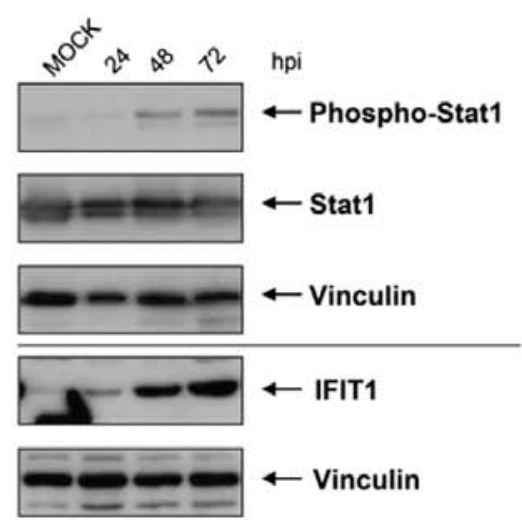

KM12

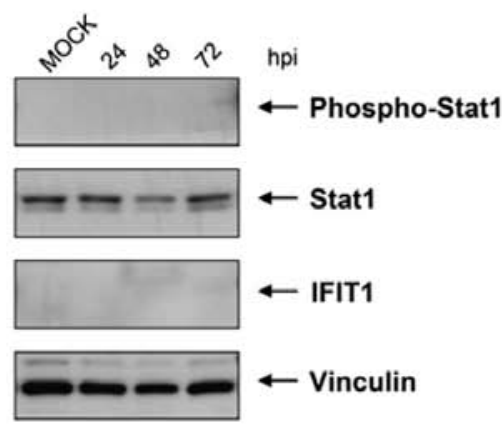

HCT-15

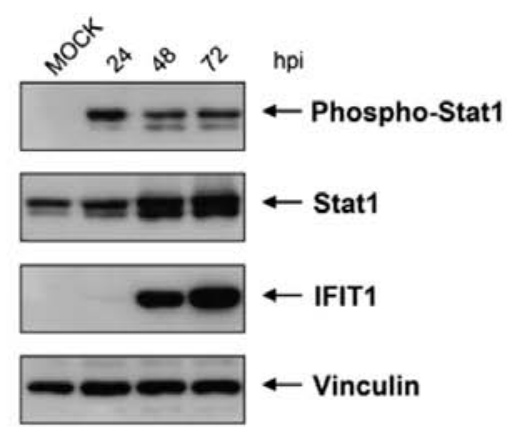

M14

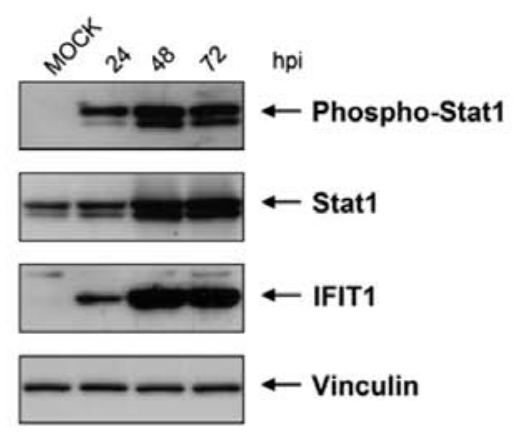

HOP-62

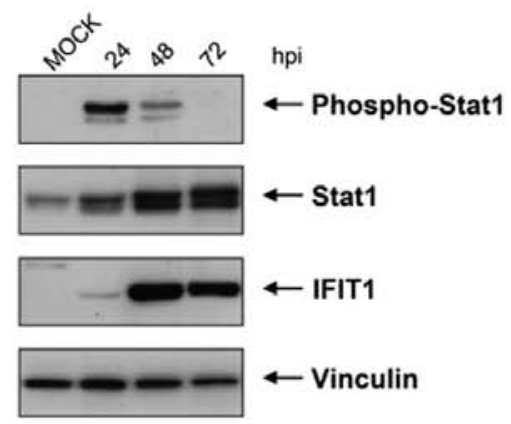

Control: MeV-SCD susceptible tumor cell line

SW-620

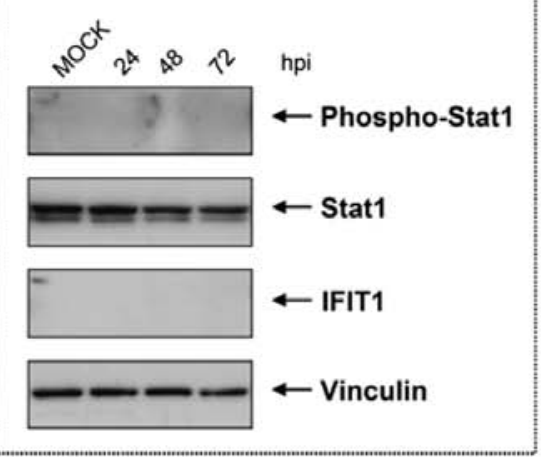

Figure 5. Induction of Stat1 phosphorylation and expression of IFIT1 after infection with MeV-SCD. Cells were infected with MeV-SCD at MOI 1 or mock infected. At the indicated time points cells were lysed. Phosphorylated Stat1, total Stat1 and IFIT1 were detected by immunoblot analysis. Vinculin was used as a loading control. Figure of cell line ACHN is composed of two separate experiments.

Overcoming oncolysis resistance phenomena to measles vaccine virus MeV-SCD by augmenting the amount of input virus and by addition of prodrug 5-FC. In order to determine the effect of using an increased MeV-SCD virus load in combination with the employment of the prodrug 5-FC on survival of the tested tumor cell lines, infections were performed not only at MOI 0.1 and 1 , but now also at MOI 10. Remnant tumor cell masses were determined again at $96 \mathrm{hpi}$.

When using an increased MOI of 10, the remaining cell masses fell below the $50 \%$ threshold for tumor cell lines ACHN, KM12 and M14, but not for HCT-15 and HOP-62 (Fig. 6). However, when additionally adding the prodrug 5-FC at 3 hpi the oncolytic effect was increased much further so that all tumor cell lines now exhibited a remnant cell mass of less than $15.50 \%$ (Fig. 6). Thus, when implementing a regimen based on an increased MOI of 10 in combination with addition of the prodrug 5-FC, resistances to 'basal' MeV-SCD-mediated oncolysis (MOI of 1; no addition of 5-FC) were successfully overcome.

\section{Discussion}

Since more and more oncolytic viruses are under clinical investigation it is by no means unexpected that such intense study activities concurrently gather more and more data on putative limitations of virotherapeutic approaches. In this context, especially both the extent as well as possible mechanisms of primary resistances to virotherapeutic vectors come into focus (16).

Oncolytic viruses are frequently able to initiate productive infections in cancer cells due to the fact that the plethora of genetic and epigenetic alterations which constitute the malignant phenotype (e.g., by promoting uncontrolled tumor cell proliferation) also could generate 'bystander' genetic/epigenetic defects in the innate immune response of transformed cells which is required to protect tumor cells from viral infection and destruction. Occasionally, cancer cells are completely devoid of antiviral activity (17), but partial inactivation is more common, leading to only limited sensitivity to oncolytic virus therapy $(9,18)$.

Measles vaccine virus ( $\mathrm{MeV})$ constitutes a leading oncolytic vector type in the field (19) encompassing four phase I trials currently actively recruiting patients exhibiting glioma, myeloma, mesothelioma and ovarian cancer. A recent detailed analysis of the clinical data obtained in the completed phase I trial of 'Intraperitoneal administration of MV-CEA, an oncolytic measles virus strain engineered to express the carcinoembryonic marker antigen, in patients with recurrent ovarian cancer' (8) revealed that i) $\mathrm{MeV}$ infected only few ovarian cancer cells; and, importantly, ii) was not substantially amplified in the tumor cells of the respective ovarian cancer patients (9). In order to overcome these limitations, it seems 
ACHN

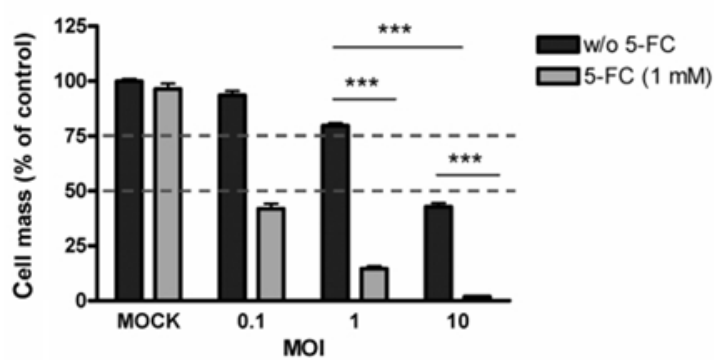

HOP-62
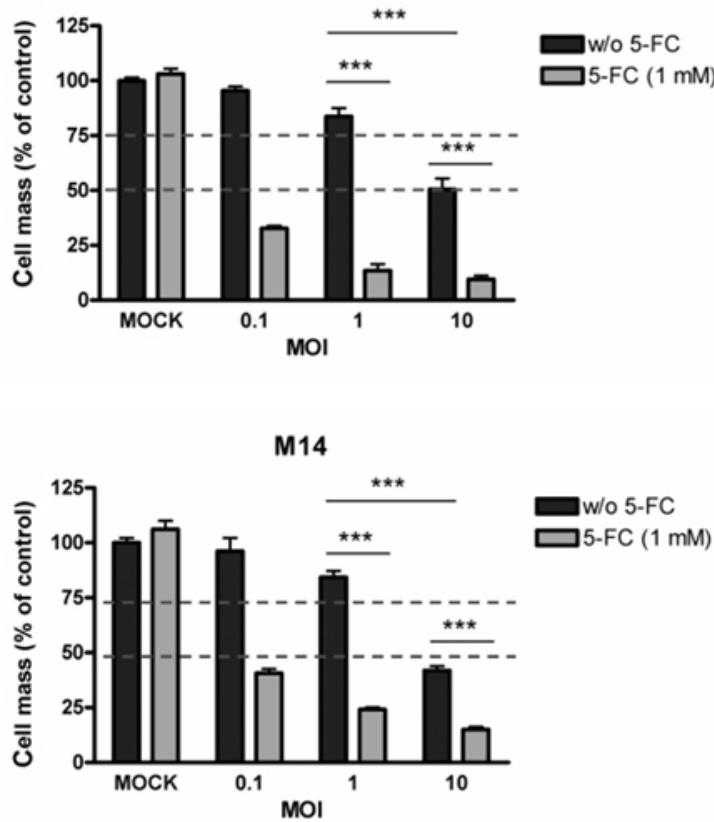

HCT-15
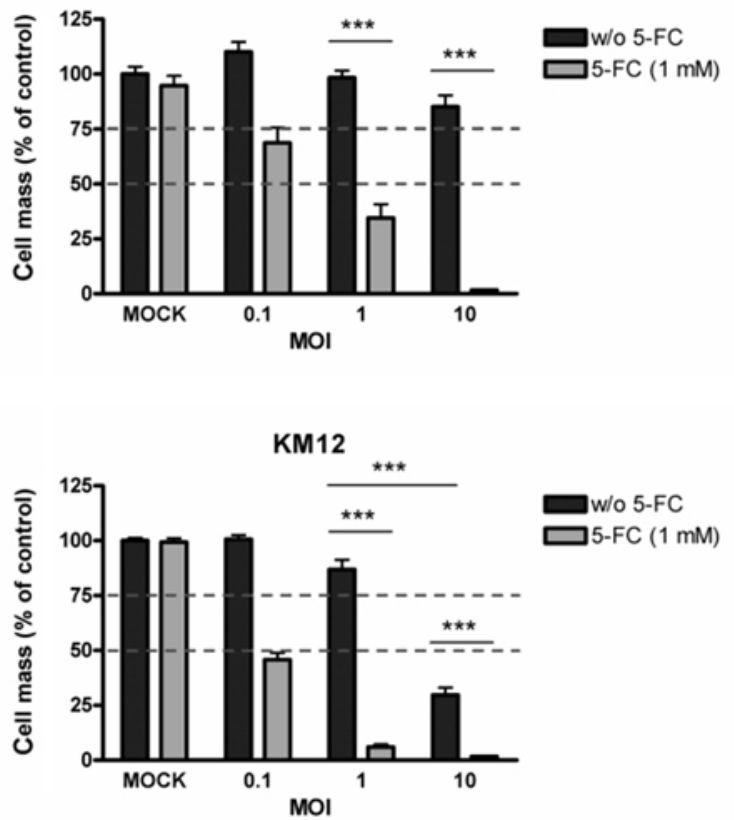

Control: MeV-SCD susceptible tumor cell line

SW-620

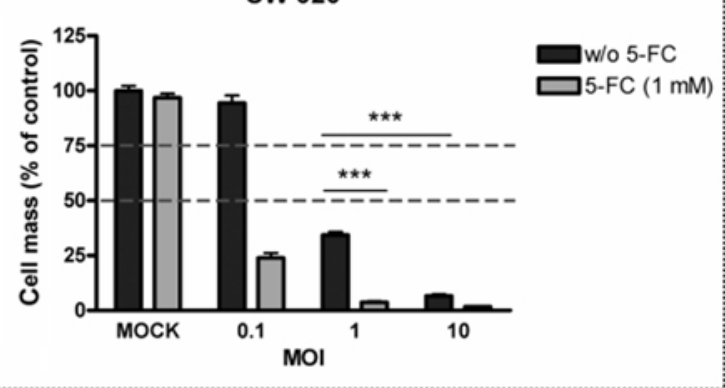

Figure 6. Cell mass of five high grade resistant and one susceptible tumor cell line $96 \mathrm{hpi}$ with MeV-SCD at different MOIs with or without addition of the prodrug 5-FC. Cells were infected with MeV-SCD at the indicated MOIs or mock infected. 3 hpi prodrug 5-FC $(1 \mathrm{mM})$ was added. Cell mass was determined 96 hpi by SRB assay. Dotted lines in red highlight the 50 and $75 \%$ remaining cell mass. ${ }^{* * * *} \mathrm{p}<0.001$.

to be required to further characterize and unravel the underlying mechanisms of primary resistance to $\mathrm{MeV}$-mediated virotherapy.

Here, we established a test platform for $\mathrm{MeV}$ and other virotherapeutics which employs the well-characterized NCI-60 tumor cell panel (10) enabling an extensive characterization of frequent/important solid tumors such as colon, CNS, lung, mammary, melanoma, ovarian, prostate and renal cancers. According to our newly defined criterion for resistance to $\mathrm{MeV}$-based virotherapeutics (remaining tumor cell mass at 96 hpi when employing a MOI of 1), 21 cell lines turned out to be partially resistant (remnant tumor cell mass $>50 \%$ ) and six cell lines were shown to be high grade resistant (remnant tumor cell mass $>75 \%$ ). Accordingly, 'only' half of the 54 tested cell lines $(n=27)$ were found to be susceptible to $\mathrm{MeV}$-mediated oncolysis, showing a remarkable decrease in cell mass (remnant tumor cell mass $<50 \%$ ) upon infection with our prototypic suicide gene armed virotherapeutic vector MeV-SCD used in this study. Importantly, upon addition of the prodrug 5-FC (thus exploiting the suicide function of vector $\mathrm{MeV}-\mathrm{SCD}$ ) all tumor cell lines exhibited a remaining cell mass of $<50 \%$, thus classifying the complete NCI-60 tumor cell panel as susceptible to MeV-SCD-mediated suicide gene therapy under the condition of now actively exploiting the arming function of MeV-SCD.

We next studied different aspects of resistance to $\mathrm{MeV}$-mediated virotherapy in our newly identified five high grade resistant tumor cell lines and in one susceptible control tumor cell line. First, we checked for possible low primary infection rates that could explain a failure in primary colonization of high grade resistant tumor cell lines. Primary infection rates (evaluated at $24 \mathrm{hpi}$ ) of high grade resistant tumor cells were found to range between 2.99 and $26.44 \%$ at MOI 1. When using an MOI of 10, much higher infection rates could be reached in all tumor cell lines (range 28.10 to $73.73 \%$ ), except for melanoma cell line M14 which was found to achieve only $6.55 \%$ GFP-positive cells at 24 hpi. Of note, all high grade resistant tumor cell lines were demonstrated to exhibit high $\mathrm{MeV}$ receptor (CD46) densities. Thereby, any primary inaccessibility of our high grade resistant tumor cells 
due to insufficient numbers of CD46 receptor proteins could be excluded.

As another aspect, host cell innate virus defense mechanisms, potentially hindering or limiting virus replication, might play a dominant role among the postulated diverse phenomena of oncolysis resistance to $\mathrm{MeV}$. While the susceptible control cell line SW-620 was demonstrated to support profound virus replication, high grade resistant $\mathrm{MeV}$-treated tumor cell lines showed different patterns of altered viral growth. In detail, two different patterns were found: i) viral growth, reaching a plateau as early as $48 \mathrm{hpi}$, indicating a reduction of viral growth, and ii) transient viral growth, reaching a peak titer at 48 hpi followed by decreasing viral titers. Western blot analysis confirmed expression of $\mathrm{MeV}$ $\mathrm{N}$-protein and SCD protein in all tested tumor cell lines. Of note, the results of our protein expression analysis were found to be concordant with the results obtained from the viral growth curves. The susceptible control cell line SW-620 which was found to exhibit a strong viral protein expression also showed a profound growth of progeny viral particles. Cell lines HCT-15 and HOP-62, which only showed a transient viral growth, also displayed a decrease in virus protein expression at $72 \mathrm{hpi}$.

In order to correlate these differences in viral replication with mechanisms of innate immunity for virus defense, we next investigated important steps of the IFN signaling pathway in the course of $\mathrm{MeV}$-based virotherapy. Indeed, high grade resistant tumor cell lines exhibiting transient viral growth were found to strongly express IFIT1 from 48 hpi on, thus perfectly correlating with the viral growth curves. Since expression of IFIT1 is known to function as a strong inducer of an active antiviral state of host cells, this parameter could be instrumental also for the characterization of virotherapeutics other than $\mathrm{MeV}$.

Another reason for resistance to $\mathrm{MeV}$ virotherapy might be constituted by malfunction of cytoplasmic sensing molecules RIG-I (retinoic acid inducible gene I) and MDA-5 (melanoma differentiation-associated gene 5) which play a key role in identifying infections with RNA viruses (20). Both intracellular receptors are involved in recognition of $\mathrm{MeV}(21,22)$. Activation of these sensing molecules by single-stranded RNAs and double-stranded RNAs respectively, leads to an activation of transcription factors such as IRF3, IRF7 or NF- $\mathrm{B}$, which then induce expression of type I IFN genes (IFN- $\alpha$ and IFN- $\beta$ ) (23). Secreted IFN binds to IFNAR-receptors, activating the Jak/STAT pathway (24) which eventually leads to expression of IFN-stimulated genes, thus inducing an efficient antiviral state of the (tumor) cell. We studied the expression of the antiviral IFN-stimulated gene (ISG) IFIT1 after infection with $\mathrm{MeV}-\mathrm{SCD}$ in five high grade resistant tumor cell lines and one susceptible control tumor cell line. Generally, activation of innate immune receptors IFNAR (IFN- $\alpha / \beta$ receptor) and of TLR3 or $\mathrm{RIG-I/MDA-5}$ leads to transcription of ISG56/IFIT1 family genes. IFIT1 expression can be triggered by IFN $-\alpha / \beta$ or IFN $-\gamma$ and by some DNA and RNA viruses. On the one hand, it can be activated via ISGF-3, consisting of IRF-9 and STAT1 and STAT2, being part of the Jak/STAT signaling pathway. On the other hand it can also be triggered directly (independently of IFN-action) by RIG-I-/MDA-5-mediated activation of IRF-3 which recognizes an IFN-stimulated response element (ISRE) in the promoter of IFIT1 and initiates expression of IFIT1. IFIT1 inhibits translation via interaction with eIF3 (25). In our study, alterations in IFN response were observed (Fig. 5). Although Stat1 was found to be expressed in all tested tumor cell lines, the high grade resistant tumor cell line KM12 as well as the susceptible control tumor cell line SW-620 showed no IFN response at all. Tumor cell lines HCT-15 and HOP-62 showed strong expression of IFIT1 at $48 \mathrm{hpi}$, which might be associated with the transient virus replication observed in these cell lines which peaked as early as $48 \mathrm{hpi}$.

In summary, we showed that MeV-SCD is a potent oncolytic vector which reliably lyses many tumor entities in vitro. Few tumor cell lines have been found to exhibit a high grade resistance to $\mathrm{MeV}$-mediated oncolysis. Fortunately, this resistance could be overcome by increasing the MOI of the virotherapeutic vector $\mathrm{MeV}-\mathrm{SCD}$ and by making use of its SCD suicide gene function. We found that in case of high grade resistance to $\mathrm{MeV}-\mathrm{SCD}$ there is no key mechanism but rather lots of cell-specific mechanisms involved in the innate immune response in tumor cells generating resistance. Taking into account the abundance of genetic and epigenetic alterations in malignant cells this finding is in favour of arming the virotherapeutic vectors, e.g. with potent suicide genes.

\section{Acknowledgements}

This study was supported in part by grants from the Deutsche Forschungsgemeinschaft (German Research Foundation, SFB 773-Project C3, U.M.L.) and by the Bundesministerium für Bildung und Forschung (German Federal Ministry of Education and Research, grant 01GU0503, U.M.L.).

\section{References}

1. Bluming AZ and Ziegler JL: Regression of Burkitt's lymphoma in association with measles infection. Lancet 2: 105-106, 1971.

2. Gross S: Measles and leukaemia. Lancet 1: 397-398, 1971.

3. Mota HC: Infantile Hodgkin's disease: remission after measles. Br Med J 2: 421, 1973.

4. Pasquinucci G: Possible effect of measles on leukaemia. Lancet 1: $136,1971$.

5. Zygiert Z: Hodgkin's disease: remissions after measles. Lancet 1: 593, 1971.

6. Parato KA, Senger D, Forsyth PA and Bell JC: Recent progress in the battle between oncolytic viruses and tumours. Nat Rev Cancer 5: 965-976, 2005.

7. Peng KW, TenEyck CJ, Galanis E, Kalli KR, Hartmann LC and Russell SJ: Intraperitoneal therapy of ovarian cancer using an engineered measles virus. Cancer Res 62: 4656-4662, 2002.

8. Galanis E, Hartmann LC, Cliby WA, et al: Phase I trial of intraperitoneal administration of an oncolytic measles virus strain engineered to express carcinoembryonic antigen for recurrent ovarian cancer. Cancer Res 70: 875-882, 2010.

9. Russell SJ, Peng KW and Bell JC: Oncolytic virotherapy. Nat Biotechnol 30: 658-670, 2012.

10. Shoemaker RH: The NCI60 human tumour cell line anticancer drug screen. Nat Rev Cancer 6: 813-823, 2006.

11. Berchtold S, Lampe J, Weiland T, et al: Innate immune defense defines susceptibility of sarcoma cells to measles vaccine virus based oncolysis. J Virol 87: 3484-3501, 2013.

12. Anderson BD, Nakamura T, Russell SJ and Peng KW: High CD46 receptor density determines preferential killing of tumor cells by oncolytic measles virus. Cancer Res 64: 4919-4926, 2004.

13. Kärber G: Beitrag zur kollektiven Behandlung pharmakologischer Reihenversuche. Naunyn-Schmiedeberg's Archives of Pharmacology 162: 480-483, 1931. 
14. Spearman C: The method of 'right and wrong cases' ('constant stimuli') without Gauss's formulae. Br J Psychol 2: 227-242, 1908.

15. Skehan P, Storeng R, Scudiero D, et al: New colorimetric cytotoxicity assay for anticancer-drug screening. J Natl Cancer Inst 82: $1107-1112,1990$.

16. Ascierto ML, Worschech A, Yu Z, et al: Permissivity of the NCI-60 cancer cell lines to oncolytic Vaccinia Virus GLV-1h68. BMC Cancer 11: 451, 2011

17. Stojdl DF, Lichty BD, tenOever BR, et al: VSV strains with defects in their ability to shutdown innate immunity are potent systemic anti-cancer agents. Cancer Cell 4: 263-275, 2003.

18. Haralambieva I, Iankov I, Hasegawa K, Harvey M, Russell SJ and Peng KW: Engineering oncolytic measles virus to circumvent the intracellular innate immune response. Mol Ther 15 588-597, 2007

19. Msaouel P, Dispenzieri A and Galanis E: Clinical testing of engineered oncolytic measles virus strains in the treatment of cancer: an overview. Curr Opin Mol Ther 11: 43-53, 2009.
20. Kato H, Takeuchi O, Sato S, et al: Differential roles of MDA5 and RIG-I helicases in the recognition of RNA viruses. Nature 441: 101-105, 2006.

21. Berghall H, Siren J, Sarkar D, et al: The interferon-inducible RNA helicase, mda-5, is involved in measles virus-induced expression of antiviral cytokines. Microbes Infect 8: 2138-2144, 2006.

22. Ikegame S, Takeda M, Ohno S, Nakatsu Y, Nakanishi Y and Yanagi Y: Both RIG-I and MDA5 RNA helicases contribute to the induction of alpha/beta interferon in measles virus-infected human cells. J Virol 84: 372-379, 2010.

23. Takeuchi $\mathrm{O}$ and Akira S: MDA5/RIG-I and virus recognition. Curr Opin Immunol 20: 17-22, 2008.

24. Darnell JE Jr, Kerr IM and Stark GR: Jak-STAT pathways and transcriptional activation in response to IFNs and other extracellular signaling proteins. Science 264: 1415-1421, 1994.

25. Fensterl V and Sen GC: The ISG56/IFIT1 gene family. J Interferon Cytokine Res 31: 71-78, 2011 